\title{
First report of CFTR mutations in black cystic fibrosis patients of southern African origin
}

S Carles, M Desgeorges, A Goldman, R Thiart, C Guittard, C A Kitazos, T J L de Ravel, A T R Westwood, $M$ Claustres, $M$ Ramsay

Laboratoire de Biochimie Genetique, CNRS UPR-9008, CRBM U249 and GREPAM, Institut de Biologie, Montpellier,

France

M Desgeorges

C Guittard

M Claustres

Department of Human Genetics, The South African Institute for Medical Research and School of Pathology, University of the Witwatersrand, PO Box 1038,

Johannesburg 2000 , South Africa

A Goldman

T J L de Ravel

M Ramsay

Division of Human Genetics, Department of Obstetrics and Gynaecology, University of Stellenbosch, Tygerberg, South Africa

R Thiart

Department of Paediatrics, Faculty of Medicine, University of Pretoria, Pretoria, South Africa

C A Kitazos

Department of Paediatrics and Child Health, Red Cross War Memorial Children's Hospital, Cape Town, South Africa A T R Westwood

Correspondence to: Dr Ramsay.

Received 5 February 1996 Revised version accepted fo publication 9 May 1996

\begin{abstract}
Cystic fibrosis (CF) is thought to be rare in the black populations of Africa who have minimal white admixture. Only a few cases have been reported but have not been studied at the molecular level. We report the detection of CFTR mutations in three southern African black patients. One was homozygous for the $3120+$ 1G $\rightarrow$ A mutation, while the other two were compound heterozygotes each with this mutation on one chromosome. The other mutations were G1249E and a previously unreported in frame 54 bp deletion within exon 17a involving nucleotides 3196-3249 (3196del54). The $3120+1 G \rightarrow A$ mutation was first described in American black patients and has been shown to be a common mutation in this population $(9-14 \%$ of CF chromosomes). It was also found in a black CF patient whose father, the $3120+1 G \rightarrow A$ carrier, is from Cameroon. These data suggest that it is an old mutation which accounts for many of the CFTR mutations in African blacks.

(7 Med Genet 1996;33:802-804)
\end{abstract}

Key words: cystic fibrosis; African blacks; CFTR mutations.

Cystic fibrosis (CF) is common in white populations of European descent, with an incidence of about 1 in 2000 , but has rarely been described in African populations with minimal white admixture. Among the mixed ancestry population of South Africa, it occurs in roughly 1 in 12000 live births. ${ }^{1}$ The incidence of CF in North American blacks has been estimated at 1 in $14000,{ }^{2}$ but the incidence in African blacks without white admixture is unknown. Since 1954, sporadic cases of CF have been reported in African blacks ${ }^{3-7}$ and there has been the impression that $\mathrm{CF}$ is underdiagnosed because of the high prevalence of confounding diagnoses including malnutrition, chronic pulmonary infections, and, more recently, tuberculosis.

This is the first report of CFTR mutations in black patients with no known white admixture. The chiefdoms of the parents are as follows: patient 1, both parents Venda; patient 2, mother Sotho, father Tswana; patient 3, mother Xhosa, father of unknown chiefdom. The clinical diagnosis of CF was made in these three cases by repeat positive sweat tests and symptoms compatible with and suggestive of CF. None had meconium ileus and all were pancreatic insufficient and had chronic lung infections. All had sputum cultures positive for Pseudomonas aeruginosa and two were positive for Staphylococcus aureus. By using PCR followed by denaturing gradient gel electrophoresis (DGGE) and sequencing of aberrant migrating or heteroduplex bands, the CFTR gene was scanned exon by exon. The methodology described by Fanen $e t a l^{\beta}$ and Claustres et al was used with minor modifications. Altered mobilities were observed for PCR products containing exons $16,17 \mathrm{a}$, and 20 from the three patients (fig 1A). Direct sequencing of the PCR products showed two known CFTR mutations $(3120+1 \mathrm{G} \rightarrow \mathrm{A}$ and G1249E) and a previously unreported mutation (3196del54).

The $3120+1 \mathrm{G} \rightarrow \mathrm{A}$ mutation in intron 16 , a splice site mutation that affects the invariant $G$ at position +1 of the donor site (Macek et al, in press), was identified in four of the six CFTR genes. The remaining CFTR genes had the G1249E mutation, a missense mutation owing to a $\mathrm{G}$ to A transition at nucleotide 3878 in exon $20^{10}$ and the deletion mutation, $3196 \mathrm{del} 54$. The $54 \mathrm{bp}$ deletion affects a region of exon 17a, starting at nucleotide 3196 and predicting the loss of 18 amino acid residues (from codon 1022 to codon 1039) in the 10th transmembrane domain (TM10) of the CFTR protein (fig 1B).

The $3120+1 \mathrm{G} \rightarrow \mathrm{A}$ mutation, found on 0.67 of CF chromosomes in this study, was first described in three American black patients (Macek et al, in press) and was also found in patient 4 , one of two black CF patients in a cohort of $200 \mathrm{CF}$ patients from Montpellier, southern France. This patient's father (the $3120+1 \mathrm{G} \rightarrow \mathrm{A}$ carrier) is from Cameroon, and his mother (the $\Delta \mathrm{F} 508$ carrier) is of mixed ancestry (black, Martinique Island and white, Brittany). CF associated haplotypes were constructed within the three South African families and in the Cameroonian family using 11 markers (table 1). The $3120+1 \mathrm{G} \rightarrow \mathrm{A}$ mutation has been shown to be common in American blacks $(14 / 112 \mathrm{CF}$ chromosomes reported to the CF Genetic Analysis Consortium ${ }^{11}$ ) and since this mutation has only been detected in black patients one can assume that it arose in Africa. Since the $3120+1 \mathrm{G} \rightarrow \mathrm{A}$ mutation has been found in association with the same haplotype in southern Africa and in central west Africa (Cameroon) it is suggested that the 
Table 1 Haplotypes associated with the CFTR mutations in the three South African black patients and one Cameroonian patient

\begin{tabular}{|c|c|c|c|c|c|c|c|c|c|c|c|c|c|}
\hline \multirow[b]{2}{*}{ Patient } & \multirow{2}{*}{$\begin{array}{l}\text { Parental } \\
\text { origin }\end{array}$} & \multirow[b]{2}{*}{ Mutation * } & \multicolumn{11}{|c|}{ Haplotype† } \\
\hline & & & met & XV & $\mathrm{KM}$ & D9 & J44 & $6 a$ & 470 & 1898 & 2694 & 3601 & 3.11 \\
\hline 1 & Mother & 3120 & 1 & 1 & 2 & 2 & 1 & 7 & 1 & 2 & 2 & 2 & 2 \\
\hline \multirow[t]{2}{*}{2} & Mother & 3120 & 1 & 1 & 2 & 2 & 1 & 7 & 1 & $2 \ddagger$ & 2 & 2 & 2 \\
\hline & Father & G1249E & 1 & 1 & 2 & 2 & 1 & 7 & 1 & 1 & 1 & 1 & 1 \\
\hline \multirow[t]{2}{*}{3} & Mother & 3120 & 1 & 1 & & & 1 & 7 & 1 & 2 & 2 & 2 & 2 \\
\hline & Father & 3196 & & 1 & & & 1 & 7 & 1 & 1 & 1 & 2 & 2 \\
\hline $4 \S$ & Father & 3120 & & 1 & 2 & & 1 & 7 & 1 & 2 & 2 & 2 & \\
\hline
\end{tabular}

$\star 3120=3120+1 \mathrm{G} \rightarrow \mathrm{A} .3196=3196 \mathrm{del} 54$.

+ Met $=$ MetH $/$ MspI; XV $=$ XV-2c/TaqI; KM $=$ KM.19/PstI; D9 = pMP6d9/MspI; J44 = J44/XbaI; 6a $=(\text { gatt })_{\mathrm{n}}$ in IVS6a; $470=\mathrm{M} 470 \mathrm{~V}$ in exon $10 ; 1898=$ $1898+152 \mathrm{~T} / \mathrm{A} ; 2694=2694 \mathrm{~T} / \mathrm{G} ; 3601=3601-65 \mathrm{C} / \mathrm{A} /$ HinfI in IVS18; $3.11=\mathrm{pJ} 3.11 / P_{s t \mathrm{I}}$.

$\ddagger$ Italics show when the phase could not be determined.

$\int$ Patient $4=$ Cameroonian CF patient.

mutation has a single origin and that it arose before the Bantu expansion roughly 2000 years ago. ${ }^{12}$ Now that a common black CF mutation has been identified, extensive carrier testing in unaffected people would give an estimate of the true frequency of $\mathrm{CF}$ in Africans.
This work was funded by the French Association against Cystic Fibrosis (AFLM) and by the South African Institute for Medical Research. Dr D W C Jacobs and Dr J C Opperman provided further clinical details on the patients.

1 Hill ID, Macdonald WBG, Bowie MD, Ireland JD. Cystic fibrosis in Cape Town. $S$ Afr Med 7 1988;73:147-9.

2 FitzSimmons SC. The changing epidemiology of cystic fibrosis. F Pediatr 1993;122:1-9.

\section{A DGGE}

Exon 16

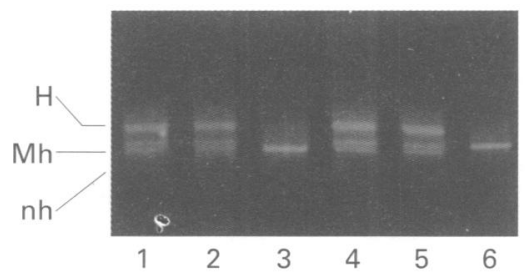

B Sequence
Exon 20

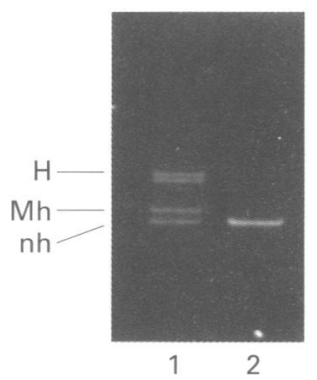

Exon 17a

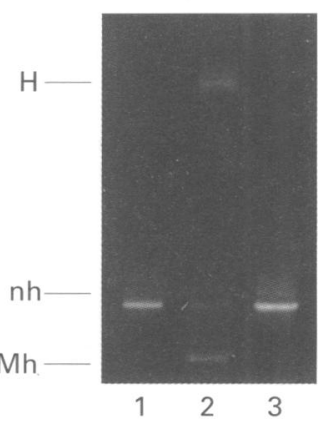

5' tTgtTATtAattgtgattggagctatAgCAgttgtcGCAgttT

TACAACCCTACATCTTTGTTGCAACAGTGCCA / GTGATAGTGGC

TtTTATTATGTTGAGAGCATATTTCCTCCAAACCTCACAGCAA )

CTCAAACAACTGgAATCTGAAg 3'

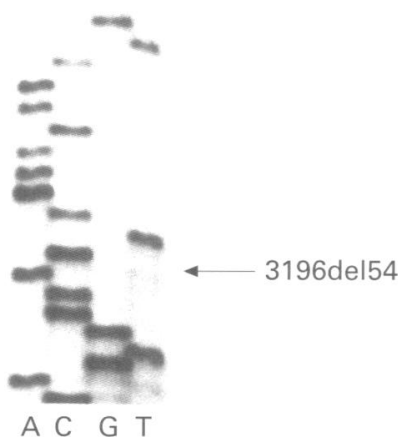

Figure 1 Characterisation of the 3196 del54 mutation. (A) Denaturing gradient gel electrophoresis patterns for CFTR exons 16, 17a, and 20. For the mutations $3120+1 G \rightarrow A$ and $G 1249 E$, the homoduplex of the mutated allele is situated at a higher position in the gel than the normal homoduplex because of lower stability owing to the replacement of $G$ by $A$ in the mutant (H: heteroduplexes; nh: normal homoduplexes; Mh: mutant homoduplexes). Exon 16: lanes 1 and 2, patient 3 and her mother (heterozygous for $3120+1 G \rightarrow A$ ); lane 3 , normal sample; lanes 4 and 5, patient 2 and his mother (heterozygous for $3120+1 G \rightarrow A$ ); lane 6, patient 1 (homozygous for $3120+1 G \rightarrow A$ ). Exon 20: lane 1, patient 2 (heterozygous for G1249E); lane 2, normal control. Exon 17 a: lanes 1 and 3, normal controls; lane 2, patient 3 (heterozygous for 3196del54). (B) Sequence context of the 54 bp deletion causing 3196del54. The deleted sequence breakpoints are shown by brackets. For the sequence analysis the mutant band was excised from an agarose gel and the DNA eluted and purified on a SpinX(Costar) column before sequencing. 
3 Grovè SS. Fibrocystic disease of the pancreas in the Bantu. $S$ Afr 7 Lab Clin Med 1959;5:113-19.

4 S Afr L Lab Clin Med 1959;5:113-19. Levin SE, Blumberg H, Zamit R, Schmaman A, Path MC Wagstaff L. Mucoviscidosis (cystic fibrosis of the pancreas) in Bantu twin neonates. $S$ Af Super M. Cystic fibrosis in southern Africa. $S$ Afr Med 1978;54:18-21.

6 MacDougall LG. Fibrocystic disease of the pancreas in African children. Lancet 1962;ii:409-10

7 McColley SA, Rosenstein BJ, Cutting GR. Differences in expression of cystic fibrosis in blacks and whites. Am 7 Dis Child 1991;145:94-7.

8 Fanen $\mathrm{P}$, Ghanem N, Vidaud M, et al. Molecular characterization of cystic fibrosis: 16 novel mutations identified by zation of cystic fibris: 16 novel mistions membrane regulator (CFTR) coding regions and splice site junctions. Genomics 1992;13:770-6.

9 Claustres M, Laussel M, Desgeorges M, et al. Analysis of the 27 exons and flanking regions of the cystic fibrosis gene: 40 different mutations account for $91.2 \%$ of the mutan alleles in southern France. Hum Molec Genet 1993;2: 1209-13.

10 Greil I, Wagner K, Rozenkranz W. A new missense mutation G1249E in exon 20 of the CFTR gene. Hum Hered 1994 44:238.

11 The Cystic Fibrosis Genetic Analysis Consortium. Population variation of common cystic fibrosis mutations. Hum Mutat 1994;4:167-77.

12 Nurse GT, Weiner JS, Jenkins T. The peoples of southern Africa and their affinities. Oxford: Clarendon Press, 1985. 